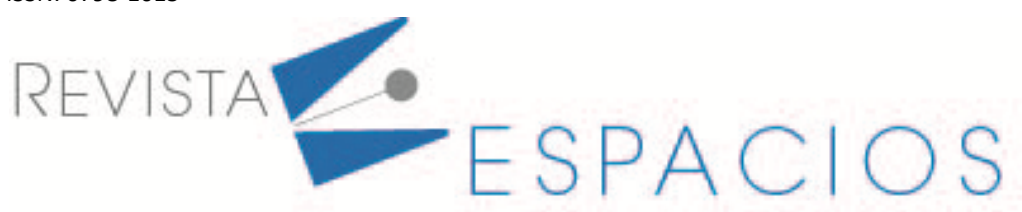

\title{
Cultura del ahorro financiero en las familias de la ciudad de Florencia (Colombia)
}

\section{Culture of financial savings in the families of the city of Florencia (Colombia)}

\author{
BERNAL, Francisco, R. ${ }^{1}$ \\ GONZÁLEZ, Gloria, L. ${ }^{2}$ \\ LOSADA, Heider, F. $^{3}$
}

\begin{abstract}
Resumen
Este artículo pretende dar a conocer la factibilidad económica para incrementar y fortalecer el ahorro financiero en las familias de la ciudad de Florencia (Colombia); se busca conocer qué tanto la población conoce sobre la cultura del ahorro, si tienen hábitos o si cuentan con asesorías para que cada uno de ellos mejore su calidad de vida económica y la de todo su núcleo familiar; igualmente, conocer todos los beneficios y servicios que han obtenido con el ahorro.

palabras clave: economía, ahorro, factibilidad, financiera.
\end{abstract}

\begin{abstract}
This article aims to present the economic feasibility to increase and strengthen financial savings in families in the city of Florencia (Colombia); It seeks to know how much the population knows about the culture of saving, if they have habits or if they have advice so that each of them can improve their quality of economic life and that of their entire family nucleus; likewise, know all the benefits and services that they have obtained with the savings.

key words: economy, savings, feasibility, financial.
\end{abstract}

\section{Introducción}

Durante la última década el comportamiento del ahorro en la ciudad de Florencia, Departamento de Caquetá de la República de Colombia, ha tenido cambios significativos; es importante explorar este campo, ya que este comportamiento ha afectado a todos los niveles de ahorro y el comportamiento financiero de la población en general. En Florencia se han realizado importantes esfuerzos dirigidos a entender y resolver la falta de acceso a servicios financieros en general, desde su inclusión dentro de las estrategias de erradicación de la pobreza extrema, hasta el diseño de una política pública de largo plazo dirigida a lograr el acceso a dichos servicios por parte de la población en general. El acceso a toda la gama de lo mencionado con anterioridad, es decir ahorro, crédito, transferencias, pagos y seguros, permite a las personas compensar los efectos de los eventos adversos que reducen sus ingresos y deterioran su calidad de vida. Se pretende hacer entender a los empleados que el mundo de la economía y las finanzas no es solo para los ricos ni los empresarios, si no que cada persona pueda manejar este tema e implementarlo en la vida cotidiana.

\footnotetext{
${ }^{1}$ Jefe de Programa Administración Financiera. Facultad de Economía y Administración. Universidad Surcolombiana. Francisco.bernal@usco.edu.co

${ }^{2}$ Docente. Facultad Economía y Administración. Universidad Surcolombiana. Gloria.gonzales@usco.edu.co

${ }^{3}$ Docente Magisterio Colombia. Heiderlosadal@hotmail.com
} 
Por tal razón, ahorrar debe convertirse en una cultura que garantice a la sociedad estabilidad económica, manteniendo un constante crecimiento, otorgando tranquilidad en tiempos difíciles como los que se están viviendo y los que se aproximan debido a la crisis económica, generada por el mismo sistema.

Desde esta postura, se justifica el presente estudio porque permitirá construir conocimiento en torno a las posibilidades económicas que tienen las familias para desarrollar una cultura del ahorro que les permita acceder a los beneficios que ofrece la bancarización y así poder cumplir con sus proyectos y en este sentido mejorar la calidad de vida. Igualmente, aportar conocimiento en torno a las estrategias de ahorro, los facilitadores y obstaculizadores del mismo, para finalmente contribuir a través de unas estrategias pertinentes al fortalecimiento de la educación financiera como herramienta para la superación de la pobreza.

\section{Sustrato teórico}

La normatividad aplicable al sector financiero está organizada jerárquicamente así: en primer lugar, en la Constitución Política de Colombia; en segundo lugar, las leyes marco expedidas por el Congreso de la República, las leyes ordinarias; en tercer lugar los decretos y reglamentos que expide el gobierno en desarrollo de las leyes marco ; en cuarto lugar las resoluciones y cartas circulares que expide el Banco de la República en el desarrollo de sus funciones, y los decretos con fuerza de ley que expide el Gobierno con base en facultades extraordinarias. Finalmente están las circulares y resoluciones que expide la Superintendencia Financiera en ejercicio de su actividad de inspección y vigilancia. Respecto a la Constitución Política Nacional, consagra en su artículo 335, que la actividad financiera, bursátil y aseguradora, en la medida en que implica el manejo, aprovechamiento e inversión de recursos de captación, es una actividad de interés público; por ello, se señala que esta actividad solamente puede ser ejercida con previa autorización del Estado, según lo establezca la ley.

En 2006, con la expedición del Conpes 3424, Colombia adoptó la política de inclusión financiera llamada "Banca de las Oportunidades". Bajo esta directriz se fomentó una intervención coordinada entre el sector público y el privado en este frente. Desde entonces el Gobierno Nacional ha introducido reformas regulatorias para facilitar la prestación de los servicios y ha entregado incentivos a las entidades financieras para promover el desarrollo de productos especializados y la adopción de metodologías de distribución ajustadas a las necesidades de la población de menores ingresos. Del lado de la industria, las entidades han participado en la implementación de la política invirtiendo importantes recursos operativos, humanos y financieros para llevar los servicios financieros a la población tradicionalmente excluida del sistema financiero.

Como antecedentes del presente estudio, se toma la investigación realizada por Melo y Zárate (2006) en donde se presenta un análisis del comportamiento del ahorro de los hogares y sus determinantes, considerando una perspectiva a largo plazo para el período 1950-2004, y otra a corto plazo a nivel microeconómico, utilizando la información de las encuestas de calidad de vida de 1997 y 2003. A nivel agregado, con base en un análisis de cointegración, se encontró una relación de largo plazo entre la tasa de ahorro de los hogares, el PIB per cápita, los impuestos directos y una medida de profundización financiera.

A nivel microeconómico se realizó un análisis de las tasas de ahorro, agrupadas según las características socioeconómicas de los hogares y para diferentes definiciones de ahorro, considerando la inversión en capital humano y la compra de bienes durables.

Así mismo presenta un análisis del comportamiento del ahorro a partir de la hipótesis del ciclo de vida, utilizado los perfiles de ahorro por año de nacimiento del jefe del hogar. Los resultados mostraron que tanto el ingreso como el consumo registran un comportamiento de $U$ invertida, sugiriendo que para el caso colombiano no hay evidencia de que se cumpla dicha hipótesis. Finalmente, al comparar los datos de ahorro de los hogares a nivel agregado, con los datos obtenidos a partir de la información de las encuestas, se encuentran diferencias que se 
pueden explicar por razones metodológicas y por el cambio en la distribución de los ingresos y de los gastos registrados entre 1997 y 2003.

La investigación conducida por Jaramillo y Dahher (2015) realizada con una metodología cualitativa con 70 personas en seis grupos focales, inicialmente señala esta población evaluada no ahorra, pero emergen sus discursos estrategias de ahorro e identifican facilitadores y obstaculizadores, produciéndose además un efecto de aprendizaje al compartir sus experiencias. Se concluyó que sí es posible el ahorro y se reflexiona sobre la importancia de incluirlo en intervenciones sociales públicas y privadas que apuesten por la educación financiera como herramienta para la superación de la pobreza. El estudio realizado por Arcos, Zapata y Hernández (2016) en donde se analizó cómo la estrategia de combate a la pobreza de la fundación Ayú, influye en el hábito del ahorro de los niños y niñas mixtecos de Oaxaca -los "Chispitas", con datos obtenidos de noviembre de 2013 a julio de 2014. Se realizaron talleres participativos y entrevistas semi estructuradas, aplicando un cuestionario a 42 niños y niñas de diez años de edad promedio, y antigüedad como ahorradores de entre tres y siete años. El perfil socioeconómico familiar corresponde mayormente a hogares campesinos. Se concluyó que los factores que inducen al ahorro son la educación, la previsión para el futuro y las emergencias, siendo las madres quienes mayor influencia tienen en la creación de este hábito este hábito. Participar en las casas visibiliza a los niños y niñas en la localidad y los inicia en la inclusión social y financiera.

\subsection{Viabilidad económica}

La viabilidad económica se enmarca dentro de los estudios de viabilidad en los cuales es considerada como la capacidad de emprender una iniciativa de inversión de asimilarse armónicamente al medio en el que se verificarán los resultados e impactos de la intervención propuesta y del que se esperan obtener beneficios. Como se conceptualizó anteriormente es una estimación de las posibilidades que tiene la idea de negocio de generar ingresos suficientes para asegurar su funcionamiento y la realización de su actividad. Está basado en hipótesis o previsiones y por lo tanto tiene un valor orientativo para poder ayudar a tomar decisiones sobre la puesta en marcha de los proyectos empresariales. Sin embargo, es imprescindible hacer este análisis de la viabilidad económica para poder tomar la decisión de emprender. Rodés (2014) en Gestión económica de la empresa señala que el estudio de viabilidad establece las previsiones de ingresos y previsiones de gastos futuros en un plazo determinado, de forma que quede estimado el resultado, beneficio o pérdida que se espera.

\subsection{Pasos a seguir en un estudio de viabilidad}

Un estudio de viabilidad se puede organizar en distintos pasos, que se describen a continuación: - Preparación del estudio: se hace un plan detallado a seguir durante el resto del estudio.- Definición del problema: Se identifica y define el problema. - Selección de las opciones de viabilidad: se producen los planes de desarrollo generales para el proyecto. - Reunión del informe: se edita el informe en documento formal.

En un estudio de viabilidad económica requiere de:

-El análisis de procedencia de los fondos que usarán para poner en marcha el proyecto.

-Determinar en dónde y en qué son invertidos todos esos fondos de forma individual.

-Elaborar una prospección de ingresos y gastos de la actividad en los años futuros.

-Estimar cuál sería el rendimiento de todo el capital invertido. 


\subsection{El ahorro financiero}

Para la escuela Keynesiana el ahorro es el residuo del consumo y la tasa de interés es una recompensa por no atesorar la totalidad del ahorro, es un premio por renunciar a la liquidez, de forma que para esta escuela el ahorro no se relaciona funcionalmente con la tasa de interés.

Existe por otro lado la teoría del ingreso permanente y otra que se llama hipótesis del ciclo vital, ambas fueron concebidas para explicar el comportamiento del consumo pero a partir de ahí es posible inferir su punto de vista sobre el ahorro. Para la teoría del ingreso permanente (Friedman citado en López, n.d.) el consumo no es función del ingreso corriente como antes lo había planteado Keynes, sino del ingreso permanente, este ingreso permanente es definido como un promedio entre el ingreso presente y el ingreso futuro, con lo que los agentes pretenden tener consumos estables. Si cambia el ingreso, el consumo lo hará si dicho movimiento en el ingreso es percibido por los agentes como permanente, pues si los agentes lo perciben como transitorio el consumo no variará. A partir de aquí se puede deducir cómo se ve el ahorro en esta teoría: en la medida en que el ingreso corriente sea más alto que el promedio, los agentes tenderán a ahorrar la diferencia y a medida que el ingreso corriente sea más bajo que el promedio los agentes tenderán a desahorrar, financiándose con deuda (Sachs, citado en López, n.d.).

En relación con la hipótesis del ciclo vital, Modigliani (1963, citado en López, n.d.) afirma que los agentes planifican su consumo a largo plazo con el fin de asignar el consumo de la mejor manera posible para mantener estable ese consumo. Según esta visión los agentes ahorran durante las épocas de altos ingresos para desahorrar en la de bajos ingresos, de forma tal que logran un nivel de consumo estable en todos los periodos.

\subsection{Factores que influyen para poder ahorrar}

Existen factores que determinan la capacidad del ahorro y estos son los más comunes: La cantidad de ingresos: Es mayor el estímulo de ahorro para el consumidor que cree que sus ingresos futuros van a disminuir con relación a los actuales. Esto sería como una planificación del consumo y de los ingresos obtenidos.

La seguridad de los ingresos futuros: Si se tiene una noción incierta de los ingresos en el futuro, la necesidad de ahorrar es mayor que cuando esos ingresos futuros están más asegurados.

Previsión del futuro: Cuando se le da menos valor a las necesidades futuras que las actuales, lo que supone vivir más en el momento actual y despreocuparse del futuro; esto acarrea un ahorro inferior.

La evolución de los precios futuros: Si se espera que los precios de los bienes van a ser más altos en el futuro, la tendencia al ahorro será menor que si se esperan unos precios estables o unos precios inferiores.

El tipo de interés: Si los intereses generados del ahorro son altos, es probable una mayor tendencia a ahorrar que si es bajo.

El salario: dependiendo del salario que se recibe, se tiene la posibilidad de ahorrar o no. Si una persona obtiene un salario mensual bastante alto, lo más probable es que ahorre la cantidad que no gasta de su salario. Mientras más alto es el salario, hay más probabilidad de ahorro.

La inflación: este es otro aspecto muy importante, ya que mientras mayor es la inflación, menor es el ahorro.

\subsection{Tasa de ahorro privado}

Dentro de los determinantes más marcadas en de las tasas de ahorro, se ha encontrado: la edad de la población, el lugar de residencia: entre mayor es la proporción de la población que vive en áreas urbanas, menor es la tasa de ahorro privado; las posibilidades de acceso al crédito y el desarrollo de los sistemas financieros. 
Identificar la naturaleza de los gastos que realiza periódicamente, separando aquellos que son indispensables (alimentos, pagos de alquiler, etcétera) de aquellos que no lo son.

\subsection{Incremento del ahorro a partir de la administración de gastos}

Amafore (2016) en su página en línea el Economista, desde una investigación realizada en México establece que una de las soluciones para hacer frente a los hábitos de consumo desordenado, es la planeación previa del mismo. Para realizarlo recomienda seguir los siguientes pasos:

1. Identificar la naturaleza de los gastos que realiza periódicamente, separando aquellos que son indispensables (alimentos, pagos de alquiler, etcétera) de aquellos que no lo son.

2. Armar una lista del presupuesto mensual, en la que deberá asignar los montos máximos de sus gastos. Recordar que la asignación de ahorro debe ir en primera línea.

3. Evitar visitar centros comerciales y autoservicios si no tiene realmente planeado realizar una compra.

4. Las ofertas únicamente lo son si generan ahorro en los gastos que tiene programados. En otro caso, sólo representan gastos adicionales que bien podría destinar al ahorro.

5. Si logra descuentos o promociones sobre los artículos que forman parte normal de su presupuesto, dirija esa diferencia a su instrumento de ahorro.

6. Recientemente, tiendas de autoservicios han abierto la opción de ventas vía telefónica o por medio de Internet. Ésta puede ser una muy buena opción para comprar los artículos específicos que desea, evitando así comprar otros artículos no necesarios.

7. Reflexionar sobre las promociones: Es frecuente que las promociones que incluyen un descuento en porcentaje $(10 \%, 15 \%$, etcétera) sean mejores que aquellas promociones que ofrecen comprar más producto (compre dos y llévese tres).

Estas recomendaciones de alguna manera contribuirán al control de gastos innecesarios.

\section{Metodología}

La investigación se enmarcó dentro de los estudios de viabilidad económica, la cual buscó la disponibilidad de los recursos necesarios para llevar a cabo los objetivos o metas propuestas. Su enfoque fue cuantitativo por cuanto se requirió establecer datos precisos con relación a los ingresos y gastos económicos de las familias, establecer la salud financiera de las mismas, pero también comprender este fenómeno desde lo humano y lo social.

\subsection{Fuentes}

Abordar esta investigación requirió de información primaria y secundaria que provenía de fuentes fidedignas sobre el tema objeto de estudio.

Fuentes primarias. La información primaria se encontró en forma directa en el campo de investigación. Fueron los datos de primera mano, sin intermediación de ninguna clase, es la que los investigadores recogieron por sí mismos, en contacto con la realidad que se estaba observando y estudiando. Esta información la suministraron las familias de esta ciudad a través de una encuesta. Fuentes secundarias. Se realizó una revisión de libros y revistas especializadas, periódicos físicos y virtuales, relacionados con la temática abordada en el presente estudio, al igual que páginas virtuales específicamente las del banco de la República para poder obtener información. 


\subsection{Población y muestra}

Para la aplicación de este estudio la ciudad de Florencia tiene aproximadamente 178500 habitantes, entre niños, jóvenes, adultos y adultos mayores; esta población se encuentra económicamente estratificada en cero, uno, dos, tres y cuatro hasta el momento. Para un futuro se espera tener el estrato cinco donde el DANE y secretaria de planeación municipal están estudiando una nueva estratificación socioeconómica.

En relación con la economía de estas familias y de la ciudad de Florencia en general el sector comercio representa un importante rubro de su economía, destacándose por la presencia de grandes complejos comerciales como el centro comercial Gran Plaza Florencia y el centro comercial La Perdiz; pero la actividad agropecuaria es la principal, según la cifra que la alcaldía demuestra es que aproximadamente el 38,3\% se desarrollan actividades comerciales, el 9,9\% se dedica a la industria, el 29,5\% a servicios, el 1,0\% a otras actividades, 21,3 a la agricultura.

La población objeto de estudio se obtuvo extrayendo total de habitantes de la ciudad de Florencia a las personas menores de 18 años de edad; es decir que de 178500 habitantes que conforman esta ciudad se le restó 80092 habitantes correspondientes a la población menores de 18 años de edad.

La población final fue entonces de 98407 habitantes y se determinaron los siguientes parámetros:

Nivel de confiabilidad: $95 \%$

Error máximo permitido: 5\%

Población: 98407

Probabilidad de éxito: $70 \%$

Improbabilidad: $30 \%$

$$
n=\frac{z^{2} * p * q * N}{e^{2} *(N-1)+\left(z^{2} * p * q\right)}
$$

Donde:

$\mathrm{n}=$ Tamaño de la muestra

$\mathrm{N}=$ Población

$Z=$ Nivel de confiabilidad

$p=$ Probabilidad de éxito

e = error máximo permitido

$q=$ Improbabilidad

$$
\begin{aligned}
& n=\frac{95 \%^{2} * 70 \% * 30 \% * 98407}{5 \%^{2} *(98407-1)+\left(95 \%^{2} * 70 \% * 30 \%\right)} \\
& n=\quad 322
\end{aligned}
$$


El tamaño muestral fue de 322 personas, entre hombres y mujeres mayores de 18 años de edad. Esta cantidad y porcentaje además de ser suficiente fue apropiada para obtener unos datos confiables.

\section{Resultados}

La información recolectada en el segundo semestre del año 2019 a través de la encuesta se procesó desde la estadística descriptiva, representándose gráficamente los resultados, para visibilizar el comportamiento de cada una de las variables en sus diferentes aspectos. Estos datos se analizaron a la luz de los referentes propuestos. Se relacionan a continuación los elementos prioritarios dentro de la implementación de los instrumentos obteniendo los siguientes resultados:

Gráfica 1

Ocupación y oficios

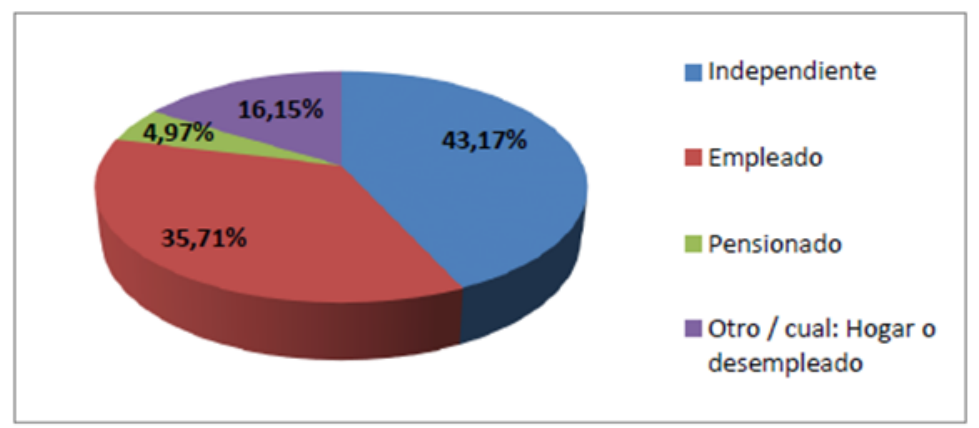

Fuente. Creación propia

Se observa qué la ocupación, oficio o profesión desempeñada por los habitantes encuestados de la ciudad de Florencia - (Colombia), marca un alto nivel la actividad como independientes con el $43,17 \%$ (ver gráfico 1), esto ayuda a establecer que es debido al desempleo que se presenta a nivel nacional y mucho más en esta ciudad tan alejada de las grandes ciudades, la falta de empresas en la ciudad, la inseguridad de los empresarios y la carencia de programas de apoyo para el emprendimiento; aunque la categoría de empleado está en el segundo lugar con un $35,71 \%$ hay que establecer mecanismos para incentivar la creación de empresas para ese 16,15\% de las personas encuestadas que por el momento se encuentran desempleados y dedicados a las actividades del hogar y para esa mayoría que se encuentran como independientes no formales.4,97\% tiene un ingreso mensual más seguro por encontrarse pensionadas. Teniendo en cuenta las actividades desempeñadas por_los encuestados se pudo observar que consolidar una cultura de ahorro toma tiempo, es necesario iniciar la tarea cuanto antes.

Gráfica 2

Fuentes de Ingreso

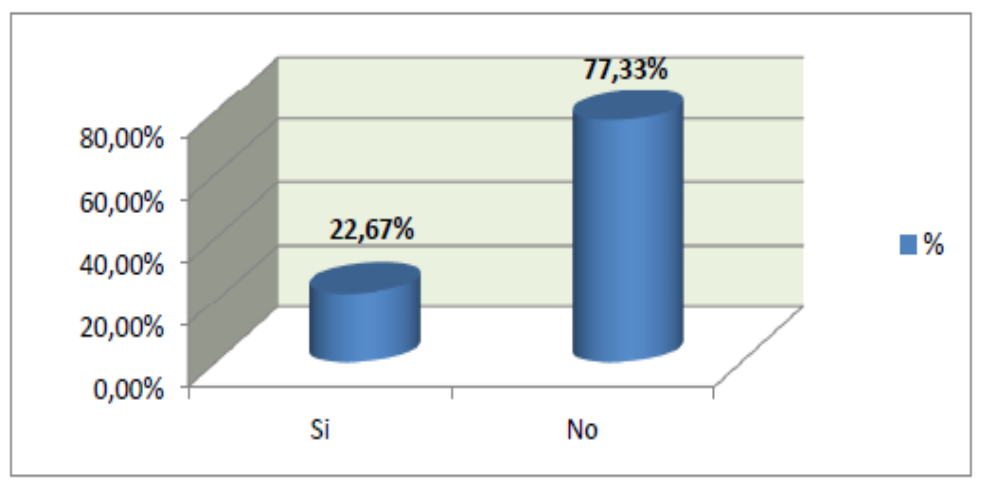

Fuente. Creación Propia 
Los resultados de las encuestas nos muestra a simple vista que el 72,98\% (Ver gráfico 2) de la población encuestada en la ciudad de Florencia no cuenta con fuentes de ingreso adicionales a su actividad desempeñada en su diario vivir (ver gráfico 3); viven de sus ingresos netos los cuales no les aportan lo suficiente para establecer una actividad adicional; este panorama es preocupante ya que tan solo el $22,67 \%$ reciben ingresos adicionales por actividades como arriendos o por ser personas prestamistas; fuera de la falta de oportunidades existe también la falta de cultura para mejorar la calidad de vida y no_solo esperar las ayudas gubernamentales. Esto demostró que la población encuestada no fomenta actividades de ahorro en su vida que les podría ayudar a solucionar los inconvenientes que se presenten.

Gráfica 3

Ingresos mensuales

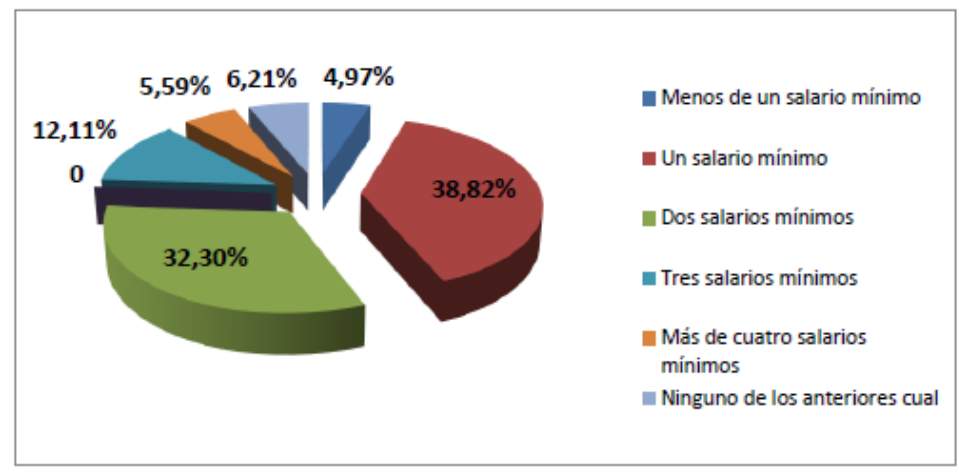

Fuente. Creación Propia

Se observar que el $47,52 \%$ de las personas encuestadas en la ciudad de Florencia cuando le sobra dinero lo gastan en otras cosas y en su momento (ver gráfica 4), no piensan en el mañana, no se pueden resistirse a sus gustos y no les gusta hacer sacrificios, el $33,85 \%$ no le sobra dinero y aunque quieran reducir a todas sus obligaciones o compromisos para que le quede dinero tendría que sacrificar cosas que no dan espera para su diario vivir, el $10,56 \%$ lo ahorra y dieron a conocer que son las personas más quisquillosas con sus gastos y tienen una disciplina más fuerte con sus ingresos, el 5,59\% lo guarda porque para ellos es necesario tener dinero reservado para alguna eventualidad, el 2,48\% lo utiliza para pagar deudas o abonar más a ellas para salir en el menor tiempo posible. La falta de cultura de ahorro ha invadido del consumismo malbaratador a la mayoría de las personas encuestadas.

Gráfica 4

Utilización del dinero

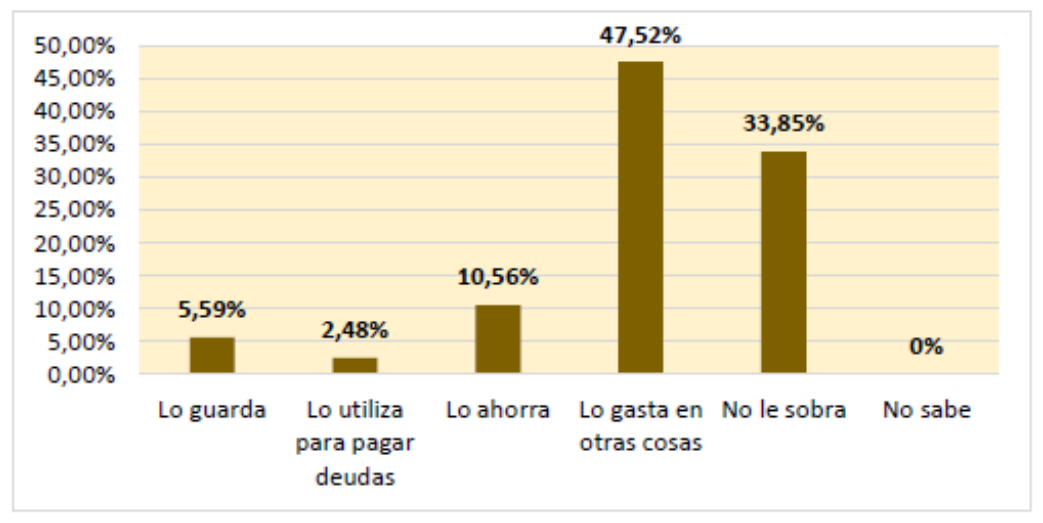

Fuente. Creación Propia

El 57,76\% de las personas encuestadas de Florencia ahorran mensualmente (Ver gráfica 5), ellos reconocen que cuando se acumulan deudas dejan de hacerlo, son muchos los que sienten que la plata se le evapora al final del 
mes y no saben qué hicieron con su dinero, el 31,06\% ahorra semanal, se pudo observar que en este caso en su mayoría son personas independientes, saben que lo tienen para financiar necesidades, imprevistos, emergencias, dificultades o para invertirles a sus negocios. El 8,07\% ahorra diariamente, se encontró en este porcentaje de personas encuestadas que eran independientes no formales, viven de su diario vivir y ahorran para soportar los momentos difíciles ya que a diario no pueden tener ni destinar dinero seguro para sus necesidades y deseos. El otro 3,11\% ahorra quincenal, son personas que reciben ingresos quincenales y son conscientes de la importancia de reservar una parte de sus ingreso de manera periódica para uso en el futuro. Se encontró que desde el hogar se debe inculcar la cultura de ahorro y transmitirse de generación en generación.

\section{Gráfica 5}

Frecuencia del ahorro

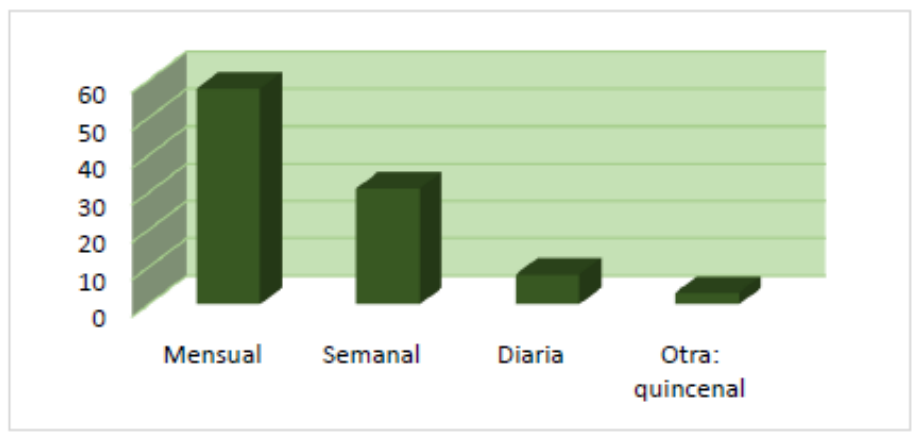

Fuente. Creación Propia

Se observa en la población encuestada de 322 habitantes se encontró una cifra alarmante de 196 personas que no ahorran (Ver gráfica 6), que corresponde al 60,87\% dieron a conocer que sus ingresos no les alcanza para hacerlo, y otros viven de lo que pueden hacer en el día, 65 personas que corresponde al 20,19\% ahorran del 1 al $20 \%$ de sus ingresos, para ellos aunque sus ingresos no sean muchos tratan en tener ahorros para cubrir emergencias, 22 personas que son el 6.83\% ahorran del 21 al 40\%, son personas que están enseñadas a conservar siempre una cifra de dinero establecida por ellos mismos y que sea constante, 15 personas que corresponde al $4,66 \%$ ahorran de 41 al $60 \%$ de sus ingresos, son personas pensionadas o que cuentan con menos obligaciones, 14 personas es decir el 4,35\% ahorran del 61 al 80 de sus ingresos, en su mayoría son independientes y aseguran ese porcentaje de dinero para momentos de crisis o para hacer futuros negocios, 10 personas que son el 3,11\% ahorran 81 al 100\% de sus ingresos ya que son personas muy mayores donde sus gastos son pocos y además tienen más ingresos adicionales. Se observó que para ser exitosos en la práctica de ahorro se debe ser realista con las finanzas y no establecer expectativas demasiado altas que no se puedan cumplir.

\section{Gráfica 6}

Ingresos destinados para el ahorro

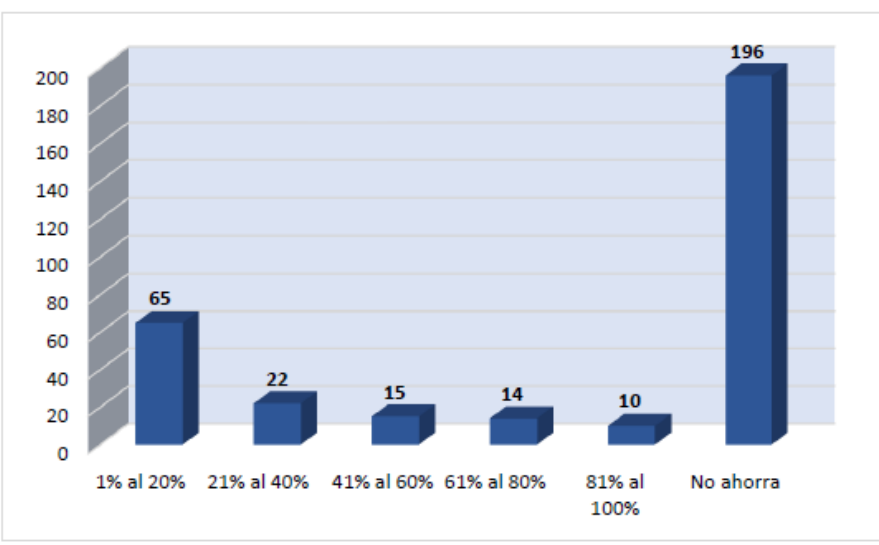

Fuente. Creación propia 


\section{Conclusiones}

En relación a los ingresos y egresos que tienen las familias de la ciudad de Florencia, se concluye que gran parte de la población tiene bajos ingresos economicos, los cuales oscilan entre estratos uno y dos por familias; asi mismo se presenta un alto nivel de empleo informal, lo cual hace que los ingresos económicos que fluctúen no sean estables para la economia.

En relación con la situación financiera de cada uno de los hogares de las familias de la ciudad de Florencia, se concluye que se presenta irregularidades, ya que no tienen una cultura del ahorro, todos sus ingresos son invertidos en sus gastos básicos, siendo esta la única proyección de inversión contrariando los planteamientos de Modigliani (1963, citado por López, nd), por el contrario se evidencia que los Habitantes encuestados tienen la tendencia de hablar del ahorro pero no realizarlo (Jaramillo y Dahher, 2015) También existe la desconfianza en las entidades financieras. La mayoría de personas piensan que ahorrar es guardar lo que les sobra de sus ingresos, sin tener en cuenta que el verdadero ahorro consiste en guardar un porcentaje mensual del salario, el cual va a contribuir para afrontar futuras crisis económicas que se pueden presentar.

Los factores que alteran el comportamiento de la tasa de ahorro financiero en las familias de la ciudad de Florencia, se concluyen que son la falta de información, educación financiera, confiabilidad y creencia en las entidades financieras; ya que no existe formación académica dirigida a este campo, conciencia ciudadana de la importancia del ahorro. Por último a la falta de las situaciones mencionadas con anterioridad se evidencia que aparte del no ahorro los ciudadanos suelen endeudarse para financiar los gastos producidos por el mal manejo de los recursos (Sachs, citado en López, n.d.).

\section{Referencias bibliográficas}

Amafore (2016) ¿Cómo administrar gastos para incrementar ahorro? El Economista. Recuperado de https://www.eleconomista.com.mx/finanzaspersonales/Como-administrar-gastos-para-incrementarahorro-20160301-0025.html

Arcos, G., Zapata, E., \& Hernández, O. (2016) Ahorro infantil “Chispitas”: Estrategia para combatir la pobreza. Texcoco, México: Colegio de Postgraduados.

Jaramillo, A y Dahher, M. (2015) El ahorro como estrategia de intervención social para la superación de la pobreza, universidades Alberto Hurtado y San Sebastián de Santiago de Chile.

López, I. (n.d.). Hipótesis del ahorro personal. En Diccionario económico. Recuperado de http://www.expansion.com/diccionario-economico/hipotesis-del-ahorro-personal.html.

Melo, J., \& Zárate, H. (2006). El ahorro de los hogares en Colombia. Revista EPE, 52, 110-161. Recuperado de http://www.banrep.gov.co/sites/default/files/publicaciones/archivos/espe_052-3.pdf.

Rodés , A. (2104). Gestión económica y financiera de la empresa. Madrid: Paraninfo. Recuperado de https://books.google.com.co/books?id=tvSwBAAAQBAJ\&printsec=frontcover\&dq=Gesti\%C3\%B3n+econ\% C3\%B3mica+de+la+empresa\&hl=es419\&sa=X\&ved=0ahUKEwj318uF8cvZAhVFt1MKHZVtA74Q6AEIJjAA\#v=onepage\&q=Gesti\%C3\%B3n\%20eco n\%C3\%B3mica\%20de\%20la\%20empresa\&f=false.

S. Soto. (11 de marzo de 2015). Cuál es la importancia de ahorrar [Mensaje en blog]. Recuperado de https://prestadero.com/blog/cual-es-la-importancia-de-ahorrar/.

Esta obra está bajo una Licencia Creative Commons Attribución-NoCommercial 4.0 International

(cc) EY-NC 